\section{Fortbildung in Managementfragen für Ärztinnen und Ärzte in leitender Stellung}

C. Zenger, J. Biaggi

Fachwissen allein genügt nicht mehr, um erfolgreich eine Klinik zu führen. Wer heute die Politik eines Spitals aktiv mitgestalten will, braucht viel nichtmedizinisches Wissen und zusätzliche Kompetenzen. Diese eignen sich einige Chefärztinnen und Chefärzte in einer längeren, systematischen Managementaus- oder -weiterbildung an. Doch nicht alle können oder wollen diese umfassende Zusatzausbildung auf sich nehmen. Viele wünschen sich ein punktuelles, problembezogenes Angebot, welches ihnen hilft, ihre spezifischen Managementaufgaben im Klinikalltag besser wahrnehmen zu können. Um diesem Bedürfnis gerecht zu werden, sind in Absprache mit der FMH Fortbildungssequenzen konzipiert worden, die exklusiv Chefärztinnen und Chefärzte sowie andere Ärztinnen und Ärzte der obersten Führungsebene ansprechen.

Wer als Ärztin oder Arzt in leitender Stellung Führungsfunktionen ausübt, kennt die Situation: Die Zeit für die eigene klinische Tätigkeit wird immer knapper. Personalfragen, Sitzungen, Budget- und Rechnungsdiskussionen, Vorsorge gegen juristische Probleme, moralische und ethisch-politische Bedenken, Kommunikationserwartungen von innen und aussen sowie die organisatorische und administrative Abwicklung all dieser Aufgaben beanspruchen einen wachsenden Anteil der eigenen Arbeits- und Freizeit. Die Umwälzungen im Gesundheitswesen verändern fortwährend die Rahmenbedingungen und verschärfen dadurch die Situation zusätzlich. Schleichend breitet sich in der Chefarztetage ein Gefühl der Verunsicherung und Resignation bei Managementfragen aus.

Korrespondenz: zenger \& biaggi Egghölzliweg 4a Postfach 418

CH-3074 Muri

E-Mail: jean.biaggi@bluewin.ch christoph.zenger@oefre.unibe.ch
Um diesem Zielpublikum kompetent eine Hilfestellung zu geben, sind Fortbildungssequenzen (ein Basiskurs sowie zusätzliche Module) konzipiert worden, die praxisbezogen und gezielt aktuelle Fragen des Führens thematisieren. Der Erfahrungsaustausch unter gleichgestellten Kollegen erhält dabei zentralen Stellenwert. Diese Führungsseminare sind Bestandteil einer umfassenden Palette von Managementfortbildungen, die mit der Unterstützung der FMH entwickelt werden sollen.

Der Basiskurs $(3 \times 2$ Tage $)$ dient der Standortbestimmung und konzentriert sich auf das eigene Führungsverhalten sowie auf Personalführungsfragen (Führungsverhalten, Teambildung und -reparatur, Gesprächsführung in schwierigen Situationen, Qualifikation und Karriereplanung für Mitarbeiterinnen und Mitarbeiter, Strategieentwicklung usw.)

Darauf abgestimmt bieten kürzere zusätzliche Module (2 bis 3 Tage) die Möglichkeit, sich dort stärker zu vertiefen, wo ein Optimierungsbedarf geortet wird. Dazu gehören namentlich:

- Verhandeln und Vermitteln;

- Betriebswirtschaft im Spital;

- Rechtsprobleme im Führungsalltag;

- Selbstmanagement;

- Krisenmanagement;

- Umgang mit Politik;

- Kommunikation und Umgang mit Medien;

- Ethik und Medizin usw.

Die Methoden sind auf die Teilnehmer zugeschnitten: Im Zentrum stehen die Interaktion und das Gespräch; Wissen wird problembezogen vermittelt. Ausgangspunkt bilden vornehmlich konkrete Probleme und Fallbeispiele der Teilnehmenden selbst. Dabei soll die eigene Situation offen mit gleichgestellten Kolleginnen und Kollegen besprochen werden können. Ausgewiesene Dozenten und Dozentinnen runden die Themen mit Hintergrundwissen und praktischen Hinweisen für den Alltag ab. Die einzelnen Module können unabhängig vom Basiskurs, d. h. auch einzeln belegt werden. Die Führungsseminare sind als Fortbildungsveranstaltungen gedacht und eignen sich für die Kreditierung durch Fachgesellschaften im Rahmen ihrer Fortbildungsprogramme.

\section{Verantwortliche Veranstalter}

Dr. iur. Christoph Zenger, Rechtsanwalt und Dozent an der Universität Bern

Dr. med. Jean Biaggi MHA, biaggi consultino, Muri bei Bern 


\begin{tabular}{lll}
\hline Die nächsten Seminare & & \\
\hline Betriebswirtschaft und Medizin & $9 .-11.4 .2002$ & Schloss Hünigen, Konolfingen \\
\hline Krisenmanagement & $2 . / 3.5 .2002$ & Studienzentrum Gerzensee \\
\hline Ethik und Medizin & $27 . / 28.6 .2002$ & Hotel Waldstätterhof, Brunnen \\
\hline Verhandeln und Vermitteln & $12 .-14.9 .2002$ & Schloss Hünigen, Konolfingen \\
\hline Medizin und Politik & $19 . / 20.9 .2002$ & Schloss Hünigen, Konolfingen \\
\hline Basismodul «Allgemeines Management im Spital» (3×2 Tage) & $\begin{array}{l}29 . / 30.8 .2002 \\
24 . / 25.10 .2002\end{array}$ & $\begin{array}{l}\text { Schloss Hünigen, Konolfingen } \\
\text { Hotel Waldstätterhof, Brunnen } \\
\end{array}$ \\
\hline
\end{tabular}

\section{Betriebswirtschaft im Spital}

Fortbildung in Managementfragen für Ärztinnen und Ärzte in leitender Stellung

Ärztinnen und Ärzte in leitender Stellung werden im Spitalalltag zunehmend mit Fragen des Finanz- und Rechnungswesens konfrontiert. Wie in jedem Unternehmen bilden diese Daten wichtige Führungsinstrumente. Oft fehlen Ärzten neben dem ungewohnten Vokabular auch die Grundbegriffe und das Verständnis für die Zusammenhänge. Dies vermittelt oft das Gefühl, dem Expertenwissen anderer ausgeliefert zu sein und erschwert das Vorbringen eigener Anliegen. Das Modul «Betriebswirtschaft im Spital» soll hier Abhilfe schaffen und Kaderleuten das entsprechende Rüstzeug geben, um als Führungsperson auch in diesen Fragen ernstgenommen zu werden.

\section{Kursinhalt}

Aufgaben und Bestandteile des Rechnungswesens; Einführung in die Bilanz und Erfolgsrechnung; Einführung in die Kostenrechnung; Technik der Kostenrechnung: Kostenstellen, Umlagen, Kostenkontrolle, Vollkosten- und Teilkostenrechnung; Ansatz der Prozesskostenrechnung, Break-even-Analyse. Ferner Planung, Budgetierung, Controlling und Reporting; Frühindikatoren und Kennziffern als Mittel der täglichen Praxis; finanzgestützte Entscheide in beson- deren Situationen wie Sortimentsfestlegung, Outsourcing, strategische Allianzen, Joint ventures, Fusionen, Schliessungen.

\section{Lernziel}

Die Teilnehmenden lernen in kleinen Gruppen und anhand von Fallbeispielen die grundlegenden Instrumente des finanziellen und betrieblichen Rechnungswesens verstehen. Sie können die daraus gewonnenen Informationen interpretieren und kritisch hinterfragen (Mitsprachekompetenz.) Sie sind mit wichtigen Teilaspekten der finanziellen Führung vertraut und haben einen geschärften Blick für finanzielle Belange. Allfällige Berührungsängste mit der finanziellen Führung sollen verschwinden.

\section{Administrative Angaben}

\section{Kursleiter}

Dr. rer. pol. Heinz Locher, Unternehmensberater, Bern Dr. rer. pol. Daniel Rentsch, Lehrbeauftragter der Universität Freiburg

Datum: 9.-11. April 2002

Kursort: Schloss Hünigen, Konolfingen

Kosten: 3 Tage inklusive Kursunterlagen: Fr. 2800.-, zusätzlich Kosten für Unterkunft und Verpflegung

\section{Anmeldung:}

zenger Ct biaggi, Frau C. Baeriswyl, Egghölzliweg 4a, Postfach 418, 3074 Muri, Tel: 03195172 34, E-Mail: jean.biaggi@bluewin.ch

Anmeldeschluss: 25. März 2002 